\title{
BMJ Open Effects of a traditional Chinese mind- body exercise, Baduanjin, on the physical and cognitive functions in the community of older adults with cognitive frailty: study protocol for a randomised controlled trial
}

Rui Xia (D) , ${ }^{1}$ Mingyue Wan, ${ }^{1}$ Huiying Lin, ${ }^{1}$ Pingting Qiu, ${ }^{1}$ Yu Ye, ${ }^{1}$ Jianquan He, Lianhua Yin, ${ }^{1}$ Jing Tao, ${ }^{2}$ Lidian Chen, ${ }^{1}$ Guohua Zheng ${ }^{3}$
To cite: Xia R, Wan M, Lin $\mathrm{H}$ et al. Effects of a traditional Chinese mind-body exercise, Baduanjin, on the physical and cognitive functions in the community of older adults with cognitive frailty: study protocol for a randomised controlled trial. BMJ Open 2020;10:e034965. doi:10.1136/ bmjopen-2019-034965

- Prepublication history for this paper is available online. To view these files, please visit the journal online (http://dx.doi. org/10.1136/bmjopen-2019034965).

RX and MW contributed equally.

Received 16 October 2019 Revised 18 March 2020 Accepted 25 March 2020

Check for updates

(c) Author(s) (or their employer(s)) 2020. Re-use permitted under CC BY-NC. No commercial re-use. See rights and permissions. Published by BMJ.

For numbered affiliations see end of article.

Correspondence to

Dr Guohua Zheng;

zhenggh@sumhs.edu.cn

\section{ABSTRACT}

Introduction Cognitive frailty (CF) is a clinical manifestation characterised by the simultaneous presence of both physical frailty and cognitive impairment among older adults without dementia and has become a new target for healthy ageing. Increasing evidence shows that regular Baduanjin (a traditional Chinese mind-body exercise) training is beneficial in improving physical function and cognitive ability in the older adults. The primary aim of this trial is to observe the effect of Baduanjin on physical and cognitive functions in older adults with CF.

Methods and analysis In this prospective, outcome assessor-blind, two-arm randomised controlled trial, a total of 102 participants with CF will be recruited and randomly allocated (1:1) into the Baduanjin training or usual physical activity control group. The control group will receive health education for $30 \mathrm{~min}$ at least once a month. Based on health education, participants in the Baduanjin exercise group will receive a 24-week Baduanjin training with $60 \mathrm{~min}$ per session and 3 sessions per week, while those in the usual physical activity control group will maintain their original lifestyle. Primary outcomes (frailty index and global cognitive ability), body composition, grip force, balance, fatigue, specific cognitive domain, including memory, execution and visual spatial abilities, and life quality of secondary outcomes will be measured at baseline, and at 13 and 25 weeks after randomisation, while the structural and functional MRI will be measured at baseline and 25 weeks after randomisation. The mixed linear model will be conducted to observe the intervention effects.

Ethics and dissemination The study has been approved by the ethics committee of the second people's hospital of Fujian province (Approval no. 2018-KL015). Results will be submitted for publication in peer-reviewed journals and disseminated at scientific conferences.

Trial registration number ChiCTR1800020341; Preresults.

\section{INTRODUCTION}

Frailty is an important syndrome resulting from the decline of multiple physiological
Strengths and limitations of this study

To our knowledge, this is the first randomised controlled trial examining the feasibility and acceptability of traditional Chinese mind-body exercise intervention in elderly with cognitive frailty (CF).

- The intervention is conducted in a local park that is easily accessible to older adults who will benefit from the familiarisation with Baduanjin programme conducted in the community to foster continuous engagement.

- This study was a prospective, outcome assessorblind, two-arm randomised controlled trial; a total of 102 participants with CF will be recruited and randomly allocated $(1: 1)$ into the Baduanjin exercise training or usual physical activity control group. It can truly reflect the intervention effect of Baduanjin on CF elders.

- In this study, MRI scans will be performed before and after the intervention. To explore the neuroimaging mechanism of Baduanjin in improving cognitive function and physical function of CF elderly.

- A methodological challenge of this study will arise from blind implementation and quality control of intervention, participants and exercise coaches in this study cannot be blinded. In order to minimise performance bias, the coaches in this study will not involve in the recruitment and outcome assessment or data analysis.

systems. ${ }^{1}$ It is often characterised by marked vulnerability to adverse health outcomes such as falls, disability and death and has become one of the biggest challenges of healthy ageing. ${ }^{2}$ Cognitive frailty (CF) is a major subtype of frailty characterised by the simultaneous presence of both cognitive impairment and physical frailty and was proposed in 2013 by the International 
Institute of Nutrition and Aging and the International Geriatrics Association. ${ }^{3}$ The prevalence of CF among the population aged over 60 years old has been reported to range from $4.4 \%$ to $9.8 \%$ in China, ${ }^{4}$ and the prevalence is up to $22 \%$ in a clinical-based survey. ${ }^{5}$ Different from simple physical frailty or cognitive impairment, CF can accelerate the cognitive and physical impairment of older adults, is more predictive for adverse health outcomes, including functional disability, low quality of life, dementia, falls and mortality, ${ }^{6}$ and has become a new target for healthy ageing. ${ }^{7}$ However, $\mathrm{CF}$ is also a reversible state, and early intervention can delay or reverse the progress of $\mathrm{CF}$ and reduce the incidence of adverse events. ${ }^{8}$

Exercise is believed to be the most effective management in older adults to improve psychological health and physical functionality. ${ }^{9}$ Several systematic reviews have indicated that regular exercise can improve the physical activity function of older adults with or without cognitive impairment. ${ }^{10-12}$ Therefore, it may be an effective measure to simultaneously improve the physical frailty and cognitive ability of older adults with CF. However, most older adults are unable to meet the recommendations from the American College of Sports Medicine and the American Heart Association for physical activity for older adults because of poor health and environmental barriers. ${ }^{1314}$ Baduanjin is a traditional Chinese qigong exercise that consists of eight movements. It is a safe aerobic exercise based on the common rules of qigong, combined with the holistic view and the theory of Qi used in traditional Chinese medicine. ${ }^{15}$ Baduanjin exercise can enhance Qi function through the whole exercise of body posture, movement, breathing and meditation, which means drawing on natural forces to optimise and balance energy within. Therefore, unlike other types of exercises, Baduanjin emphasises mindfulness and breathing adjustments while exercising the limbs and is recommended for older adults in China. ${ }^{16}$ Some systematic reviews have shown that regular practice of Baduanjin can result in physiological benefits, including increasing strength and flexibility, reducing fat and blood pressure, ${ }^{17}$ enhancing physical fitness and improving cardiovascular risk factors and mental health. ${ }^{18-21}$ In addition, Baduanjin was also reported to improve the global cognitive function, memory and executive ability of older adults with mild cognitive impairment in the community. ${ }^{22}$ Therefore, it is reasonable that Baduanjin may potentially improve the physical and cognitive functions of older adults with CF.

With the development of Morphometric MRI technology, the robust imaging biomarkers for diseases related brain damage have educed the potential for clinical impact. For example, the MRI studies have established the association of cognitive impairment and either morphometric or functional connectivity changes of brain. Previous studies reported reductions in the volumes and dysfunction in connectivity of the whole hippocampus, medial temporal lobe and entorhinal cortex in the brains of older adults with cognitive impairment compared with healthy subjects. ${ }^{23} 24$ Although few studies focus on CF, degenerations compatible with cognitive impairment have been reported. Recent imaging studies based on voxelbased morphometry (VBM) and blood oxygenation level-dependent signal analysis showed that Baduanjin exercise induced structural and functional connective changes in the human brain. The changes in grey matter (GM) volume, especially in the hippocampus, have been linked to an improved memory function. ${ }^{25}$ Analysis of seed-based resting state functional connectivity ( $\mathrm{rsFC}$ ) also showed that Baduanjin could decrease rsFC between the medial prefrontal cortex and orbital prefrontal gyrus/putamen and in the right rolandic operculum, right middle temporal gyrus, right supramarginal inferior parietal gyrus and angular gyrus ${ }^{26} 27$; in addition, Baduanjin could increase rsFC between the hippocampus and right angular gyrus. ${ }^{22}$ This suggests that Baduanjin can modulate the default mode and dorsal attention networks in older adults with or without cognitive impairment. Therefore, we can infer that the impact of Baduanjin exercise on the morphology or functional connection of related-brain region in the older adults with CF may be an important mechanism for improving CF.

To investigate the effects and mechanisms of 6-month Baduanjin training on CF, we plan to conduct a prospective, randomised controlled trial (RCT). The main aim of this trial will be to determine the effects of Baduanjin training on the cognitive and physical functions of older adults with CF. Additionally, we aimed to determine how Baduanjin exercise impacts the structural and functional connectivity of specific brain regions or brain networks using resting functional MRI scans.

We hypothesise that Baduanjin training will show improvement in the physical and cognitive functions compared with no specific exercise intervention. With neuroimaging, we hypothesise that differential structural and functional connection changes will be observed between the Baduanjin training and control groups.

\section{METHOD/DESIGN}

\section{Study design}

The design of this study is a double arm RCT in a 1:1 allocation ratio with allocation concealment and assessor blinding. A total of 102 eligible participants will be randomly assigned to the 24-week Baduanjin exercise intervention (60 min per session, three sessions per week) or the 24-week usual activity control group. The outcomes of cognitive and physical functions will be assessed at baseline, 13 weeks and 25 weeks (at the end of intervention), while the structure and function of brain regions related to cognitive and motion functions will be measured by using fMRI at baseline and 25 weeks. All data collectors will be blinded to the group assignments. The study 


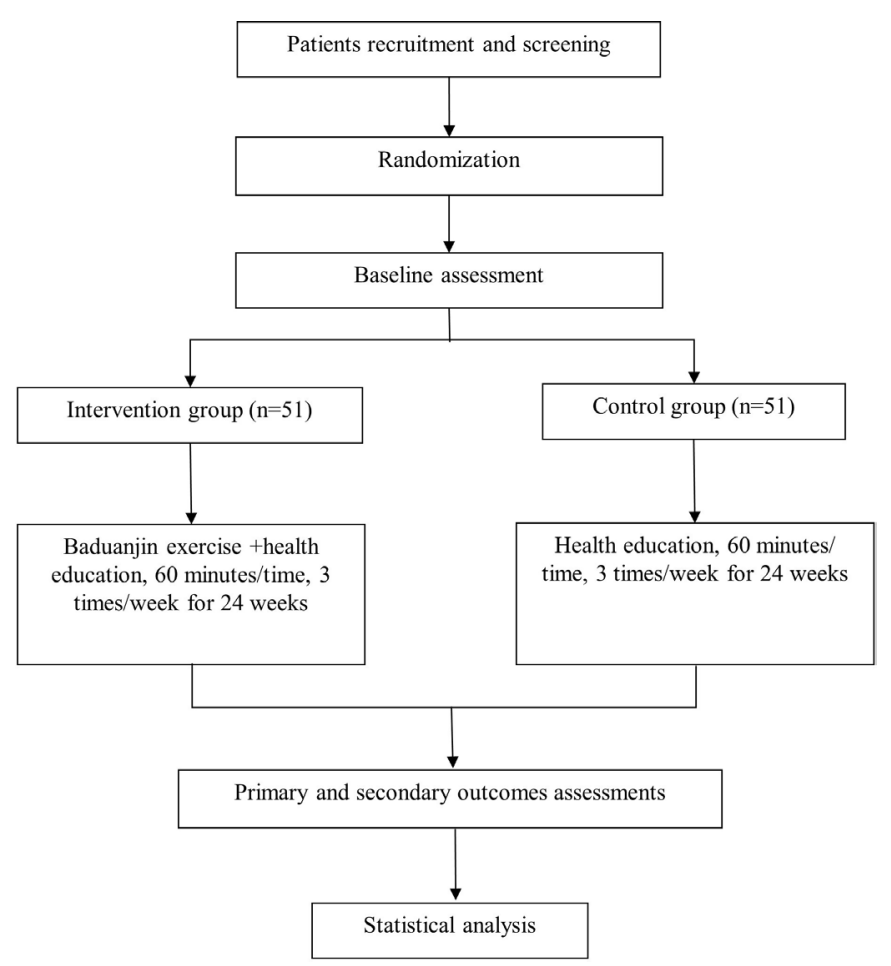

Figure 1 Flow chart of the trial.

procedure and outcome assessment schedule for this trial are presented in figures 1 and 2 .

\section{Study population}

The study population is a community older adults with CF. The diagnostic, inclusion and exclusion criteria for the study sample are as follows.

\begin{tabular}{|c|c|c|c|c|c|c|}
\hline \multirow[b]{2}{*}{ TIMEPOINT } & \multirow{2}{*}{$\begin{array}{c}\text { Screening } \\
-2 \mathrm{~W}\end{array}$} & \multirow{2}{*}{$\begin{array}{r}\text { Pre } \\
-1 \mathrm{~W}\end{array}$} & \multicolumn{3}{|c|}{ 24-week Intervention } & \multirow{2}{*}{$\begin{array}{l}\text { Post } \\
+1 \mathrm{~W}\end{array}$} \\
\hline & & & ow & $12 \mathrm{~W}$ & $24 \mathrm{~W}$ & \\
\hline \multicolumn{7}{|l|}{ ENROLMENT } \\
\hline Eligibility screen & $x$ & & & & & \\
\hline Informed consent & $x$ & & & & & \\
\hline ALLOCATION & & $x$ & & & & \\
\hline \multicolumn{7}{|l|}{ ASSESSMENTS } \\
\hline Basic characteristics & & $x$ & & $x$ & & $x$ \\
\hline Frailty Index & & $x$ & & $x$ & & $x$ \\
\hline Global cognitive function & & $x$ & & $x$ & & $x$ \\
\hline Body composition & & $x$ & & $x$ & & $x$ \\
\hline Grip force & & $x$ & & $\mathrm{x}$ & & $x$ \\
\hline Balance function & & $x$ & & $x$ & & $x$ \\
\hline Fatigue & & $x$ & & $x$ & & $x$ \\
\hline Memory & & $x$ & & $\mathrm{x}$ & & $x$ \\
\hline Execution function & & $x$ & & $x$ & & $x$ \\
\hline Visual spatial function & & $x$ & & $x$ & & $x$ \\
\hline Quality of life & & $x$ & & & & $x$ \\
\hline MRI & & $x$ & & & & $x$ \\
\hline Inflammatory biomarkers & & $x$ & & & & $x$ \\
\hline Adverse events & & & & $x$ & & $x$ \\
\hline \multicolumn{7}{|l|}{ INTERVENTIONS } \\
\hline $\begin{array}{l}\text { Intervention group (Baduanjin } \\
\text { exercise + health education) }\end{array}$ & & & & & & \\
\hline $\begin{array}{l}\text { Control group } \\
\text { (Health education) }\end{array}$ & & & & & & \\
\hline
\end{tabular}

The $\times$ indicates at which point of the trial the respective assessments will take place.

Figure 2 Schedule of enrolment, assessments and interventions.
Diagnostic criteria

According to Chang's definition of $\mathrm{CF}^{28}$ participants with CF should have: (1) Edmonton Frailty Scale (EFS)Chinese Revised $\geq 5$ points; (2) cognitive decline in accordance with age and education (Montreal Cognitive Assessment (MoCA) $\leq 26$ points); and (3) absence of dementia (Global Deterioration Scale (GDS) score of 2 or 3 ).

\section{Inclusion criteria}

The eligible participants met the following criteria: (1) diagnosed with CF; (2) aged 60 years or older; (3) had no regular physical exercise for at least half a year (regular exercise means exercise with a frequency of at least twice a week and at least 20 min per session); and (4) provided informed consent.

\section{Exclusion criteria}

The exclusion criteria were as follows: (1) patients with hypertension with uncontrollable blood pressure (systolic blood pressure greater than $160 \mathrm{~mm} \mathrm{Hg}$ or diastolic blood pressure greater than $100 \mathrm{~mm} \mathrm{Hg}$ ); (2) history of alcohol and drug abuse; and (3) history of mental illness (including personality disorder, schizophrenia, etc), major depression (Beck Depression Scale>10 points), severe aphasia and audio-visual disorders, severe organ failure, history of coronary artery disease, musculoskeletal disorders and other exercise contraindications; (4) metal implants (including fixed metal dentures, pacemakers, etc) and other reasons making the patient unsuitable for MRI scans; (5) participating in another clinical study.

\section{Withdrawal criteria and management}

Participants will be allowed or required to withdraw from the study based on the following: (1) have major violations with the study protocol; (2) suffer from a serious disease, preventing the continuation in the trial; (3) suffer from serious adverse events related to the research during the study period; and (4) voluntary request to be withdrawn from the trial.

\section{Recruitment}

Participants will be recruited from Jinniushan Community Center, Wenquan Community Center and Wufeng Community Center in Fuzhou city through a combination of online and offline efforts, including using the Internet, posting posters, sending leaflets and setting up onsite recruitment stations. The potentially eligible individuals will first complete a screening by community doctors in the Community Health Service Center to determine their eligibility according to the inclusion and exclusion criteria. The eligible individuals who are interested in participating in the study will have an informed discussion with trained research assistants. The research assistants will obtain written informed consent from individuals willing to participate in the study before starting the baseline assessment. 


\section{Randomisation, allocation concealment and blinding}

After the baseline assessment, the eligible participants will be randomly assigned to the Baduanjin exercise group or the usual activity control group. The random allocation sequences and allocation concealment will be conducted as described in detail previously. ${ }^{29}$ We cannot blind participants, exercise coaches or intervention supervisors to the assigned treatment, but outcome evaluators and data statisticians will be blinded to group allocation.

\section{Intervention}

\section{Baduanjin exercise group}

Participants in the Baduanjin exercise group will receive 24 weeks of Baduanjin exercise training with a frequency of 3 days a week and $60 \mathrm{~min}$ a day, including a 15 min warm up, 40 min Baduanjin training and 5 min cool down, and health education on nutrition and diet-related knowledge for older adults once every 4 weeks (at least $30 \mathrm{~min}$ per session). Baduanjin training will be assigned at three community centres (Jinniushan Community Center, Wenquan Community Center and Wufeng Community Center in Fuzhou city) with 15-20 individuals per centre. Three professional coaches, who have been engaged in teaching Baduanjin exercise to college students at the Fujian University of Traditional Chinese Medicine (FJTCM) for at least 5 years, will be employed to guide participants' training. The training scheme of Baduanjin exercise is planned as described in detail previously, ${ }^{29}$ and consists of 10 postures (including the preparation and ending postures; figure 3). ${ }^{30}$ A Polar heart rate (HR) monitor (Mio Sport SD) will be used to monitor participants' energy consumption and HR during training.

\section{Usual physical activity control group}

The participants in the usual physical activity control group will not receive any specific exercise training from the study scheme besides health education on nutrition and diet-related knowledge. They will be required to maintain their original activity habit.
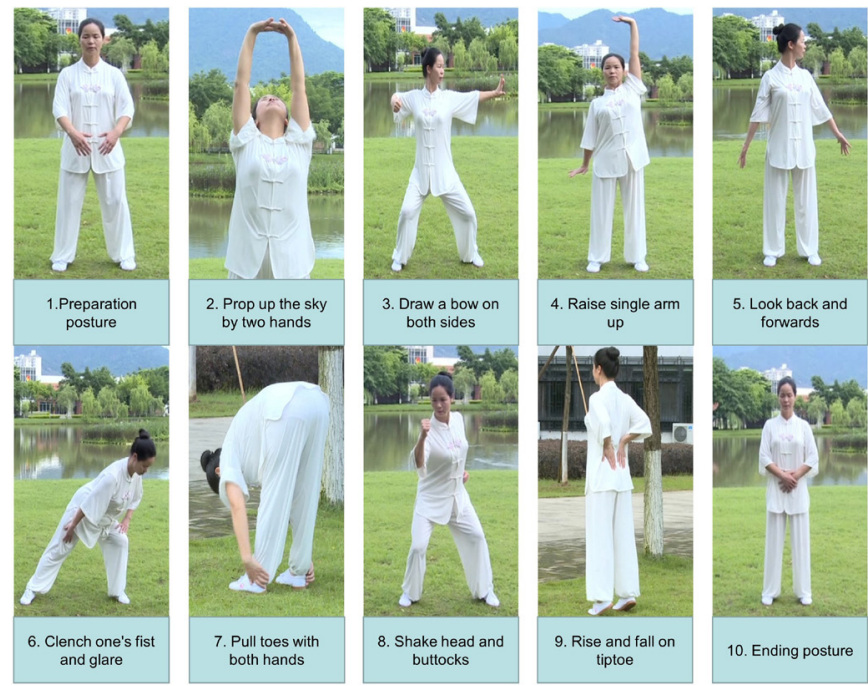

Figure 3 The postures of Baduanjin.
To exclude bias from the excess activity of participants, all participants in both the Baduanjin exercise and usual physical activity control groups will be advised not to seek any other regular exercise and will be required to record an activity during the trial period according to method described previously. ${ }^{29}$

\section{Outcome assessment}

The variables in this trial consist of basic information, primary outcomes and secondary outcomes. The basic characteristics will be measured at baseline (1-2 weeks before randomised allocation). The primary and secondary outcomes will be measured at baseline and at the end of the intervention period ( 25 weeks after randomisation). In addition, the outcomes of cognitive and physical functions will be also assessed in the middle of the intervention period (13weeks after randomisation). All primary and secondary outcomes will be assessed by the experienced staff at the Second People's Hospital Health Management of Fujian Province, who will be blinded to the allocation results of participants. A summary of all measurements in this trial is shown in figure 2.

\section{Basic information}

Participants' demographic characteristics (eg, sex, age, education, marital status, living arrangements, occupation and socioeconomic status) and history of disease and medication use will be collected by the recruiters using the self-designed questionnaire. The basic MoCA, EFS, GDS and Beck Depression Inventory will be assessed using the corresponding scales. Baseline measurements will be completed before randomisation.

\section{Primary outcomes}

The frailty index will be assessed using the Chinese version of the EFS. The EFS samples had 9 domains (ie, cognitive ability, general health status, functional independence, social support, drug use, nutrition, emotions, control and functional performance); the maximum score is 17 and represents the highest level of frailty. ${ }^{31}$

Global cognitive function will be measured using the MoCA scale, which is a cognitive screening instrument created and validated to detect mild cognitive impairment. MoCA is a brief (approximately $10 \mathrm{~min}$ ) test that evaluates visuospatial/executive functions, naming, verbal memory registration and learning, attention, abstraction, 5 min delayed verbal memory, and orientation with a total score of 0-30 (a higher score equates to a better function). The Chinese version of the MoCA (Fuzhou version) will be applied to this trial with good validity, reliability and sensitivity in the population in Fuzhou city. ${ }^{32}$

\section{Secondary outcomes}

Body composition comprises body weight, fat-free weight, body water, protein, muscle mass, fat mass, presumed bone mass, body fat rate, basic metabolism and segmental analysis of muscle mass and fat rate of trunk and limbs and will be measured using the Belida Body Fat Meter (TBF-418B), which is a bioelectrical impedance analysis. 
Grip force will be measured using an electronic grip force metre (CAMRY-EH101).

The balance function will be estimated using a dynamic and static balance assessment analyser (model: Bismarck Super Balance), and this will include the visual adjustment coefficient, balance stability coefficient and proprioceptive coefficient; in addition, the balance index of the subjects will be obtained.

Fatigue will be measured using the Fatigue Scale-14 (FS-14). The FS-14 scale comprises 14 items; items 1-8 assess body fatigue, while items 9-14 assess mental fatigue. Higher scores indicate more serious fatigue.

Memory will be evaluated using the Wechsler Memory Scale-Revised, Chinese version (WMS-RC), which can assess the long-term, short-term and instantaneous memory. The score of the WMS-RC is expressed as a memory quotient $(\mathrm{MQ})$, and a higher $\mathrm{MQ}$ means better memory.

The execution function will be measured using the Trail Making Test (TMT), which is usually divided into two parts: TMT-A and TMT-B. The test programme is as described in detail previously. ${ }^{29}$ The TMT-B/TMT-A ratio is taken as a valid index of executive function.

Visual spatial function will be measured using the ReyOsterrieth Complex Figure Test. This test consists of 18 scoring parts, and each part is given a score of $0-2$. Higher scores indicate better visuospatial ability.

Quality of life will be measured by the Medical Outcomes Study 36-Item Short-Form Health Survey (SF-36).$^{33}$ It consists of 36 items that assess 8 health concepts: functional capacity (10 items), physical activities (4 items), social activities (2 items), bodily pain (2 items), mental health (5 items), usual activities (4 items), vitality (4 items) and general health status (five items). ${ }^{34}$ Each health concept is evaluated separately by a normalised score of $0-100$, with 0 corresponding to the worst health status, and 100 corresponding to the best health status. The Chinese version of the SF-36 has been reported to have high reliability and validity in the Chinese population. $^{35} 36$

Structural MRI and resting-state functional MRI data will be collected at baseline and postintervention. An MRI scan will be conducted in the Medical Imaging Department of Rehabilitation Hospital Affiliated to FJTCM. A Siemens Prisma 3.0 T MRI system and Siemens 64-channel head and neck coil will be used for acquiring fMRI data. The scanning parameters are listed as follows:

T1: repetition time $(\mathrm{TR})=2300 \mathrm{~ms}$, echo time $(\mathrm{TE})=2.27 \mathrm{~ms}$, flip angle $=8^{\circ}$, slice thickness $=1.0 \mathrm{~mm}$, field of view $(\mathrm{FOV})=250 \mathrm{~mm} \times 250 \mathrm{~mm}$, matrix $=256 \times 256$, voxel size $=0.98 \times 0.98 \times 1 \mathrm{~mm}^{3}$, number of slices $=160$.

Resting fMRI: $\mathrm{TR}=2000 \mathrm{~ms}, \mathrm{TE}=30 \mathrm{~ms}$, flip angle $=90^{\circ}$, slice thickness $=3.6 \mathrm{~mm}, \quad \mathrm{FOV}=230 \mathrm{~mm} \times 230 \mathrm{~mm}$, matrix $=64 \times 64$, voxel size $=3.6 \times 3.6 \times 3.6 \mathrm{~mm}^{3}$, number of slices $=37$, axial slices $=35$, phases per location $=240$.

After all images are checked for artefacts and structural abnormalities, the images will be segmented into GM, white matter (WM) and cerebrospinal fluid and normalised using the high-dimensional DARTEL (diffeomorphic anatomical registration through exponential lie algebra) algorithm. The fMRI preprocessing will consist of motion correction, slice-timing correction, normalisation and Gaussian spatial smoothing. DTI (diffusion tensor imaging) sequences will be conducted for the measurement of WM hyperintensities and connectivity.

\section{Safety measurements}

Participants will be monitored during the intervention period for the occurrence of adverse events. Any adverse events, including exercise injury and fall, will be detailed and reported by the research assistant to the research group using an adverse event case report form (CRF). The causality in relation to the Baduanjin intervention and the severity of adverse events will be evaluated. Serious adverse events will be reported to the ethics committee.

\section{Sample size}

Sample size was calculated based on improvements in EFS or MoCA scores because of two primary outcomes in this trial. According to relevant published literature, ${ }^{37} 38$ the mean and SD of EFS scores after the intervention between the experimental and control groups are 7.7 (2.0) and 9.3 (2.3), respectively, and the effect size of the aerobic exercise intervention on the global cognitive function of MCI patients is approximately $0.54-0.58$, with a median value of 0.55 . A sample size of 60 or 84 participants was calculated to be enough to detect the target effect EFS or MoCA changes with a type I error of 5\% and $80 \%$ power, respectively, through Gpower V.3.1.9.2 software. To ensure the target effect of each primary outcome, we decided to use 84 participants as the basis of the sample size calculation. Considering a $20 \%$ attrition rate, a total of 102 participants is necessary, with 51 participants in each group.

\section{Statistical analysis}

Statistical analysis will be performed using the SPSS V.24.0 software package by an independent statistician, with a statistical significance of $p$ value $<0.05$ on both sides. Continuous variables will be expressed using the mean and SD for the normal distribution or median and its IQR for a non-normal distribution. Categorical variables will be described as frequencies or percentages. The missing data will be filled in using the multiple imputation method.

Primary and secondary (besides MRI data) outcomes will be analysed using the mixed-effect linear model with restricted maximum likelihood. In this model, the independent variables are the group (Baduanjin group and usual physical activity control group), time (different measurement time points) and possible confounding factors. The differences between the Baduanjin and control groups after the intervention will be analysed using Student's t-test or Mann-Whitney U test.

For the MRI data, DPABI (a toolbox for Data Processing \& Analysis for Brain Imaging, Version 2.3_170105, 
http://rfmri.org/dpabi) software package will be used to preprocess the image data obtained by MRI scans under Matlab2013a (The Match Works). The image will be transformed into a standard space by preprocessing, including slice timing, realignment, coregistration, smoothing ( $4 \mathrm{~mm}$ full width at half maximum), filtering $(0.01-0.1 \mathrm{~Hz})$, and so on; then, further group comparisons will be carried out. VBM will be used to compare the GM volume in the hippocampus and other regions of interest. Independent component analysis will be used to extract time sequence signals for core brain network components, including the default mode, central executive and salience networks. Components will be extracted from the preprocessed images using GIFT V.4.0 (http:// icatb.sourceforge.net/). Two-sample t-tests will be used to compare the differences of the functional connections of the brain network between the groups before and after the intervention. Then, we will analyse the correlation between the changes in brain network functional connections and frailty index or cognitive function.

Adverse effects (AEs) will be analysed using $\chi^{2}$ test or Fisher's exact test. If formal statistical analyses between groups cannot be performed due to the lack of power, AEs will be tabulated and summarised using descriptive statistics.

\section{Data collection and management}

Data will be collected by the outcome assessors using the CRF. Then, research assistants will transcribe the CRF into an electronic data acquisition system (EDC). This is a free Research Manager (ResMan, http://www. medresman.org) provided by the China Clinical Trial Registry and meets the available standards for security. The research data will be stored in the EDC system in a separate password-protected location.

\section{Patient and public involvement}

Before the recruitment stage, we invited a portion of the older adults in the community to investigate the research design and feasibility of the intervention measures. According to their opinions, we adjusted the intervention time to avoid schedule incompatibilities and chose what they are interested in for health education content. No patients or members of the public will be engaged in recruiting or in conducting and measuring outcomes. The results of the study will be sent to participants through a written report and email. The burden of the interventions included in this study will not be assessed by patients.

\section{Ethics issues}

This study protocol is in accordance with and was based on the Helsinki Declaration. Ethics approval was obtained from the Medical Ethics Committee of the Second People's Hospital of Fujian Province (Approval no. 2018KL015). All participants will provide voluntary written informed consent after fully discussing potential benefits and risks before participating.

\section{Dissemination}

The study protocol has been registered and can be obtained through the Chinese Registry website (registered in ChiCTR.org with the identifier ChiCTR1800020341). The results of the study will be presented at local, national or international conferences about cognitive rehabilitation and will be submitted as manuscripts to peer-reviewed journals. The main findings of this study will also be shared to all participants and disseminated to researchers, health service providers, healthcare professionals and the public through courses, demonstrations and the internet regardless of the magnitude or direction of the effects.

\section{DISCUSSION}

As one of the most common forms of traditional Chinese exercise, Baduanjin has become a popular and safe community exercise to promote health in older adults of China. An increasing number of studies have reported that Baduanjin exercise has a positive effect on improving the cognitive ability and physical function in older adults in the community. Therefore, it is reasonable to assume that regular Baduanjin training could be beneficial to cognitive and physical functions in the CF population. This study was designed to test whether Baduanjin has these effects. This trial will employ rigorous methods to control bias, including randomisation, parallel control and blinding of the outcome assessors and statisticians. To ensure that participants master standard Baduanjin movements, we will also employ qualified physical exercise teachers to serve as the Baduanjin exercise coaches. A specific and accessible training scheme $(60 \mathrm{~min}$ per day and 3 days each week for 24 weeks) will be applied as the Baduanjin intervention, making it possible to clearly clarify the association with cognitive or physical function. Furthermore, the extensive outcome assessment on several levels involving neuropsychological testing and MRI scan assessment will make it possible to explore the mechanism of action of the Baduanjin intervention on brain functional networks. The results should be helpful in improving the cognitive and physical functions of the participants with $\mathrm{CF}$ and should be generalisable to the community-dwelling older population.

In summary, this study will be the first RCT to assess the effects of Baduanjin on cognitive and physical functions in older adults with CF. If this trial is successfully conducted and demonstrates a significant result, this study will be helpful in establishing the optimal nonpharmacological treatment for CF and possible neuroimaging mechanisms. This study will also provide reliable evidence for the application of regular Baduanjin exercise in a community of older adults with CF.

\section{Author affiliations}

${ }^{1}$ College of Rehabilitation Medicine, Fujian University of Traditional Chinese Medicine, Fuzhou, China

${ }^{2}$ Rehabilitation Medicine College, Fujian University of Traditional Chinese Medicine, Fuzhou, Fujian, China 
${ }^{3}$ College of Nursing and Health Management, Shanghai University of Medicine \& Health Sciences, Shanghai, China

Acknowledgements The authors wish to thank Yan Zheng from health management centre of the Second People's Hospital of Fujian province, as well as Ming Li from the Imaging Department of Fujian Rehabilitation Hospital for assistance with the development of materials related to this trial. In addition, the authors would like to thank the community health service centres of Jinniushan, Wenquan and Wufeng in Fuzhou for their help in recruiting participants.

Contributors LC, JT and GZ conceived the study, designed the study protocol and drafted the manuscript. RX wrote the manuscript and participated in the coordination and implementation of the study. GZ wrote several sections of the manuscript and revised and finalised the study protocol. JT is in charge of coordination and direct implementation. MW, PQ, HL, YY, JH and LY helped to develop the study measures and analyses. All authors contributed to drafting the manuscript and have read and approved the final manuscript.

Funding National Natural Science Foundation of China, 81574045.

Competing interests None declared.

Patient and public involvement Patients and/or the public were involved in the design, or conduct, or reporting, or dissemination plans of this research. Refer to the Methods section for further details.

Patient consent for publication Obtained.

Provenance and peer review Not commissioned; externally peer reviewed.

Open access This is an open access article distributed in accordance with the Creative Commons Attribution Non Commercial (CC BY-NC 4.0) license, which permits others to distribute, remix, adapt, build upon this work non-commercially, and license their derivative works on different terms, provided the original work is properly cited, appropriate credit is given, any changes made indicated, and the use is non-commercial. See: http://creativecommons.org/licenses/by-nc/4.0/.

ORCID iD

Rui Xia http://orcid.org/0000-0002-5941-8263

\section{REFERENCES}

1 Clegg A, Young J, Iliffe S, et al. Frailty in elderly people. The Lancet 2013;381:752-62.

2 Fried LP, Tangen CM, Walston J, et al. Frailty in older adults: evidence for a phenotype. J Gerontol A Biol Sci Med Sci 2001;56:M146-57.

3 Kelaiditi E, Cesari M, Canevelli M, et al. Cognitive frailty: rational and definition from an (I.A.N.A./I.A.G.G.) international consensus group. $J$ Nutr Health Aging 2013;17:726-34.

4 Ma L, Zhang L, Zhang Y, et al. Cognitive frailty in China: results from China comprehensive geriatric assessment study. Front Med 2017;4:174.

5 Delrieu J, Andrieu S, Pahor M, et al. Neuropsychological Profile of "Cognitive Frailty" Subjects in MAPT Study. J Prev Alzheimers Dis 2016;3:151-9.

6 Roppolo M, Mulasso A, Rabaglietti E. Cognitive frailty in Italian community-dwelling older adults: prevalence rate and its association with disability. J Nutr Health Aging 2017;21:631-6.

7 Cano A. Cognitive frailty, a new target for healthy ageing. Maturitas 2015;82:139-40.

8 Ruan Q, Yu Z, Chen M, et al. Cognitive frailty, a novel target for the prevention of elderly dependency. Ageing Res Rev 2015;20:1-10.

9 Buford TW, Anton SD, Clark DJ, et al. Optimizing the benefits of exercise on physical function in older adults. PM\&R 2014;6:528-43.

10 Cadore EL, Rodríguez-Mañas L, Sinclair A, et al. Effects of different exercise interventions on risk of falls, gait ability, and balance in physically frail older adults: a systematic review. Rejuvenation Res 2013;16:105-14.

11 Sanders LMJ, Hortobágyi T, la Bastide-van Gemert S, et al. DoseResponse relationship between exercise and cognitive function in older adults with and without cognitive impairment: a systematic review and meta-analysis. PLoS One 2019;14:e0210036.

12 Gomes-Osman J, Cabral DF, Morris TP, et al. Exercise for cognitive brain health in aging: a systematic review for an evaluation of dose. Neurol Clin Pract 2018;8:257-65.

13 Nelson ME, Rejeski WJ, Blair SN, et al. Physical activity and public health in older adults: recommendation from the American College of sports medicine and the American heart association. Med Sci Sports Exerc 2007;39:1435-45.

14 Schutzer KA, Graves BS. Barriers and motivations to exercise in older adults. Prev Med 2004;39:1056-61.

15 Koh TC. Baduanjin -- an ancient Chinese exercise. Am J Chin Med 1982;10:14-21.

16 Zheng G, Huang M, Li S, et al. Effect of Baduanjin exercise on cognitive function in older adults with mild cognitive impairment: study protocol for a randomised controlled trial. BMJ Open 2016;6:e010602.

17 Zou L, SasaKi JE, Wang $\mathrm{H}$, et al. A systematic review and metaanalysis Baduanjin Qigong for health benefits: randomized controlled trials. Evid Based Complement Alternat Med 2017;2017:1-17.

18 Zou L, Pan Z, Yeung A, et al. A review study on the beneficial effects of Baduanjin. J Altern Complement Med 2018;24:324-35.

19 Cheng FK. Effects of Baduanjin on mental health: a comprehensive review. J Bodyw Mov Ther 2015;19:138-49.

20 Mei L, Chen Q, Ge L, et al. Systematic review of Chinese traditional exercise baduanjin modulating the blood lipid metabolism. Evid Based Complement Alternat Med 2012;2012:1-8.

21 Zheng G, Fang Q, Chen B, et al. Qualitative evaluation of Baduanjin (traditional Chinese Qigong) on health promotion among an elderly community population at risk for ischemic stroke. Evidence-Based Complementary and Alternative Medicine 2015;2015:1-10.

22 Tao J, Liu J, Chen X, et al. Mind-Body exercise improves cognitive function and modulates the function and structure of the hippocampus and anterior cingulate cortex in patients with mild cognitive impairment. Neuroimage Clin 2019;23:101834.

23 Gu L, Zhang Z. Exploring structural and functional brain changes in mild cognitive impairment: a whole brain ale meta-analysis for multimodal MRI. ACS Chem Neurosci 2019;10:2823-9.

24 Anatürk M, Demnitz N, Ebmeier KP, et al. A systematic review and meta-analysis of structural magnetic resonance imaging studies investigating cognitive and social activity levels in older adults. Neurosci Biobehav Rev 2018;93:71-84.

25 Tao J, Liu J, Liu W, et al. Tai chi Chuan and Baduanjin increase grey matter volume in older adults: a brain imaging study. J Alzheimers Dis 2017;60:389-400.

26 Liu J, Tao J, Liu W, et al. Different modulation effects of tai chi Chuan and Baduanjin on resting-state functional connectivity of the default mode network in older adults. Soc Cogn Affect Neurosci 2019;14:217-24.

27 Xia R, Qiu P, Lin H, et al. The effect of traditional Chinese Mindbody exercise (Baduanjin) and Brisk walking on the dorsal attention network in older adults with mild cognitive impairment. Front Psychol 2019;10:10

28 Won CW, Lee Y, Kim S, Chang W, Yunhwan L, Sunyoung K, et al. Modified Criteria for Diagnosing "Cognitive Frailty". Psychiatry Investig 2018;15:839-42.

29 Zheng G, Zheng Y, Xiong Z, et al. Effect of Baduanjin exercise on cognitive function in patients with post-stroke cognitive impairment: study protocol for a randomised controlled trial. BMJ Open 2018;8:e020954.

30 Zheng G, Li M, Lan X, et al. The effect of Baduanjin exercise for physical and psychological wellbeing of college students: study protocol for a randomized controlled trial. Trials 2013;14:422.

31 Yang L, Bao L, Zhou Q, et al. Reliability and validity of Edmonton frail scale. Int J Geriatr 2016;37:135-7.

32 Fang $\mathrm{Y}$, Tao $\mathrm{Q}$, Zhou $\mathrm{X}$, et al. Patient and family member factors influencing outcomes of poststroke inpatient rehabilitation. Arch Phys Med Rehabil 2017;98:249-55.

33 Qu B, Guo HQ, Liu J, et al. Reliability and validity testing of the SF-36 questionnaire for the evaluation of the quality of life of Chinese urban construction workers. J Int Med Res 2009;37:1184-90.

34 Ware JE, Sherbourne CD. The mos 36-Item short-form health survey (SF-36). Med Care 1992;30:473-83.

$35 \mathrm{Jin}$ W, Yu H. A study of the reliability and validity of SF-36 scale on evaluating health of population. Chin Health Resour 2012;15:265-7.

36 Lam CLK, Tse EYY, Gandek B, et al. The SF-36 summary scales were valid, reliable, and equivalent in a Chinese population. J Clin Epidemiol 2005;58:815-22.

37 Tarazona-Santabalbina FJ, Gómez-Cabrera MC, Pérez-Ros P, et al. A multicomponent exercise intervention that reverses frailty and improves cognition, emotion, and social networking in the community-dwelling frail elderly: a randomized clinical trial. J Am Med Dir Assoc 2016;17:426-33.

38 Song D, Yu DSF, Li PWC, et al. The effectiveness of physical exercise on cognitive and psychological outcomes in individuals with mild cognitive impairment: a systematic review and meta-analysis. Int $J$ Nurs Stud 2018;79:155-64. 